\title{
Correlação entre a altura e as dimensões das pregas vocais
}

\section{Correlation between height and vocal folds dimensions}

\author{
João Aragão Ximenes Filho ${ }^{1}$, Erich Christiano \\ Madruga de $\mathrm{Melo}^{2}$, Christiano de Giacomo \\ Carneiro', Domingos Hiroshi Tsuji ${ }^{3}$, \\ Luiz Ubirajara Sennes ${ }^{4}$
}

\section{Palavras-chave: estatura, laringe, cordas vocais, anatomia, histologia. \\ Key words: body height, larynx, vocal cords,} anatomy, histology.

\section{Resumo / Summary}

E $\mathrm{m}$ algumas situações clínicas ou durante procedimentos cirúrgicos sobre 0 arcabouço laríngeo, faz-se importante o conhecimento preciso das dimensões das pregas vocais. Objetvo: Avaliar a existência de correlação entre as características dos indivíduos estudados como altura, peso, idade e sexo com as dimensões macroscópicas da porção membranosa das pregas vocais. Forma do Estudo: Experimental. Métodos: Foram estudados 24 cadáveres do Serviço de Verificação de Ó bito entre Abril e Outubro de 2001, sendo 13 homens e 11 mulheres. De cada indivíduo, foram obtidos os dados de altura, peso e idade com posterior remoção da laringe. Foi utilizado paquímetro digital para as medidas das dimensões das pregas vocais. Resultados: Não foram encontradas diferenças entre as pregas vocais direita e esquerda quanto ao comprimento $(p=0,91)$, largura $(p=0,93)$ ou espessura $(p=0,72)$. As pregas vocais masculinas foram maiores $(p<0,001)$ e mais largas $(p=0,04)$ que as femininas, não havendo diferença quanto à espessura $(p=0,11)$. Foi observada uma forte correlação $(r=0,76)$ entre a altura dos indivíduos e o comprimento das pregas vocais. Não houve correlação entre altura e largura $(r=0,36)$ ou espessura $(r=0,39)$ das pregas vocais. Conclusões: As pregas vocais masculinas foram maiores e mais largas do que as femininas. 0 comprimento da porção membranosa das pregas vocais variou em função da altura dos indivíduos.

\begin{abstract}
I. n some clinical situations or during laryngoplastic procedures, it is important the knowledge of the dimensions of the vocal folds. Aim: The aim of this study was to evaluate the correlation existence among the individuals' characteristics studied as height, weight, age and sex with the macroscopic dimensions of the membranous portion of the vocal folds. Study Design: Experimental. Methods: We studied 24 cadavers (13 men and 11 women) submitted to autopsy from April and October of 2001. Of each individual, we obtained height, weight and age with subsequent removal of the larynx. We used digital pachimetry for the measures of the dimensions of the vocal folds. Results: We did not find difference between the vocal folds right and left in length $(p=0,91)$, width $(p=0,93)$ or thickness $(p=0,72)$. The masculine vocal folds were larger $(p<0,001)$ and wider $(p=0,04)$ that the feminine ones, and it did not have difference between the thickness $(p=0,11)$. We observed a strong correlation $(r=0,76)$ between the individuals' height and the length of the vocal folds. We did not find correlation between height and width $(r=0,36)$ or thickness $(r=0,39)$ of the vocal folds. Conclusions: Males vocal folds were larger and wider than females'. The length of the membranous portion of the vocal folds varied in function of the individuals' height.
\end{abstract}

${ }^{1}$ Doutor em Otorrinolaringologia pela Faculdade de Medicina da Universidade de São Paulo (FMUSP). Médico Assistente (voluntário) do Serviço de O torrinolaringologia do Hospital Universitário da Faculdade de Medicina da Universidade Federal do Ceará ${ }^{2}$ Doutorando da Disciplina de O torrinolaringologia da FMUSP.

${ }^{3}$ Prof. Livre Docente e Responsável pelo Grupo de Voz da Divisão de Clínica O torrinolaringológica do Hospital das Clínicas da FMUSP. ${ }^{4}$ Prof. Livre Docente e Associado da Disciplina de Otorrinolaringologia da FMUSP e Diretor do Serviço de Bucofaringolaringologia do Hospital das Clínicas da FMUSP. Endereço para Correspondência: João Aragão Ximenes Filho - R. Paula Ney, 700 60140-200 Fortaleza CE Tel (0xx85) 268-3641 - E-mail: joaoximenesf@bol.com.br

Trabalho realizado na Disciplina de O torrinolaringologia da Faculdade de Medicina da Universidade de São Paulo (FMUSP) e apresentado no $36^{\circ}$ Congresso Brasileiro de Otorrinolaringologia, Florianópolis-SC, Novembro de 2002 Artigo recebido em 20 de janeiro de 2003. Artigo aceito em 24 de março de 2003. 


\section{INTRODUÇÃO}

Nas últimas décadas tem aumentado o número de procedimentos cirúrgicos sobre 0 arcabouço laríngeo. Cirurgias para medialização de uma prega vocal paralisada como a tireoplastia tipo I de Isshiki ${ }^{1}$, para mudança da freqüência fundamental como as tireoplastias tipo III e IV ${ }^{1}$, técnicas para correção de insuficiência glótica ${ }^{2,3}$ e para avanço de comissura anterior $r^{3,4}$ somente foram possíveis com 0 avanço nos conhecimentos sobre anatomia, histologia e fisiologia da laringe.

Anatômica e fisiologicamente, as laringes de indivíduos do gênero masculino e feminino têm características diferentes, que repercutem no seu padrão vibratório ${ }^{2}$. Sabese que 0 ângulo entre as lâminas da cartilagem tiróide é maior em mulheres e que as pregas vocais são mais compridas nos homens ${ }^{2}$. No entanto, ainda são pouco conhecidas as variações individuais nas dimensões das pregas vocais entre indivíduos do mesmo gênero.

Assim, o objetivo deste estudo foi avaliar a existência de correlação entre as características dos indivíduos estudados como altura, peso e idade com as dimensões macroscópicas da porção membranosa das pregas vocais. Enfoque especial foi dado na comparação entre a laringe masculina e feminina a fim de observar eventuais diferenças.

\section{CASUÍSTICA E MÉTODOS}

\section{Casuística}

0 projeto de pesquisa deste estudo foi aprovado pela Comissão de Ética para Análise de Projetos de Pesquisa (CAPPesq) do Hospital das Clínicas da Faculdade de Medicina da Universidade de São Paulo em Abril de 2001, permitindo a realização do mesmo, bem como a obtenção de peças do Serviço de Verificação de Óbitos da Capital (SVOC) da Universidade de São Paulo.

Foram estudados 24 cadáveres sendo 13 de indivíduos do sexo masculino e 11 do sexo feminino, no período de Abril a Outubro de 2001. A idade variou de 29 a 90 anos, com média de 62,2 e desvio padrão de 17,4. Com relação à raça, 10 eram brancos, 9 pardos, 4 negros e 1 indivíduo da raça amarela. Todos residiam na cidade de São Paulo e foram vítimas de morte não-violenta e de causas não relacionadas com a laringe. Excluímos aqueles que tenham sido submetidos previamente à traqueotomia, cirurgia laríngea ou radioterapia na região cérvico-facial.

\section{MÉTODOS}

\section{Obtenção da laringe}

Após seleção dos cadáveres, foi realizada incisão na pele da região torácica alta e levantamento de retalho cutâneo tóraco-cervical. A laringe foi removida em bloco após secção sobre o osso hióide e no nível do quarto anel traqueal. No laboratório, foi realizada a dissecção da laringe.

\section{Estudo morfométrico}

$\mathrm{Na}$ ausência de anormalidades macroscópicas ao exame com lupa, foram realizadas as medidas macroscópicas abaixo citadas, dividindo os parâmetros em dois grupos:

1. Características dos indivíduos - onde foi verificado sexo, idade, altura e peso dos cadáveres. Para a obtenção da altura foi utilizada fita métrica, enquanto para o peso os dados foram anotados das informações contidas no prontuário do cadáver no Serviço de Verificação de Ó bitos a partir de dados fornecidos por seus familiares;

2. Características macroscópicas das pregas vocais - onde foi medido o comprimento, a largura e a espessura da porção membranosa das pregas vocais, em milímetros com o auxílio de um paquímetro apropriado, sendo a largura e a espessura medidas a meia distância entre a comissura anterior e o processo vocal da cartilagem aritenóide.

As medidas macroscópicas das dimensões da porção membranosa das pregas vocais foram realizadas pelo mesmo pesquisador de forma a evitar vícios. As medidas foram todas realizadas com o mesmo paquímetro e todas na mesma seqüência: comprimento e largura, sendo posteriormente realizada abertura posterior da laringe para a medida da espessura (dimensão supero-inferior das pregas vocais). 0 comprimento foi medido desde a inserção da prega vocal na cartilagem tireóide, inferiormente ao pecíolo da epiglote até a sua inserção no processo vocal da cartilagem aritenóide. A largura foi definida como a distância compreendida entre a borda livre e a transição entre o ligamento vocal e o assoalho do ventrículo laríngeo. A espessura foi medida após a incisão longitudinal da cartilagem cricóide, entre as cartilagens aritenóides, com o mínimo de abertura possível para permitir a entrada do paquímetro e expressa a dimensão crânio-caudal das pregas vocais. Foi definida como a distância entre dois planos paralelos traçados superior e inferiormente ao músculo tireoaritenóide.

\section{Análises realizadas}

As análises realizadas foram divididas em:

1. Características dos indivíduos, comparando as médias obtidas nos grupos masculino e feminino.

2. Comparação entre as médias das dimensões macroscópicas dos lados direito e esquerdo das pregas vocais.

3. Correlação entre o sexo e as características macroscópicas das pregas vocais.

4. Correlação entre as características dos indivíduos com as dimensões das pregas vocais.

\section{Análise estatística}

Foi realizado teste de Kolmogorov-Smirnov para análise da distribuição dos dados coletados. Uma vez que 
obtivemos distribuição gaussiana, foram empregados testes paramétricos como o teste $t$ de Student nas comparações quanto ao sexo e entre as médias. Foi pesquisada a existência de correlação entre as características dos indivíduos e as respectivas dimensões de suas pregas vocais utilizando 0 Coeficiente de Correlação de Pearson. Foi considerado índice de significância de $95 \%(p<0,05)$. 0 programa estatístico utilizado foi o SPSS versão 10.0 para Windows.

\section{RESULTADOS}

\section{Características dos indivíduos}

A idade no grupo masculino foi de 58,7 $\pm 18,7$ anos, enquanto no grupo feminino foi de $66,4 \pm 15,6$ anos. Comparando os dois grupos, não houve diferença estatística $(p=0,29)$. A média das alturas foi de $170,2 \pm 8,9 \mathrm{~cm}$, sendo de $176,5 \pm 6,1 \mathrm{~cm}$ no sexo masculino e de $162,6 \pm 4,7 \mathrm{~cm}$ no sexo feminino $(p<0,001)$. O grupo masculino foi em média $13,9 \pm 2,25 \mathrm{~cm}$ mais alto que 0 grupo feminino. $A$ média do peso foi de $70,5 \pm 11,7 \mathrm{~kg}$, sendo $71,5 \pm 11,8 \mathrm{~kg}$ no sexo masculino e $69,5 \pm 12,1 \mathrm{~kg}$ no sexo feminino $(p=0,69)$. 0 Gráfico 1 mostra a comparação dessas variáveis nos dois grupos.

\section{Comparação entre os lados direito e esquerdo}

A análise comparativa entre os lados direito e esquerdo não mostrou diferença quanto ao comprimento $(p=0,91)$, largura $(p=0,93)$ ou espessura $(p=0,72)$. Também não mostrou diferença estatística quando analisamos os lados direito e esquerdo em função dos grupos masculino e feminino. Assim, para cada indivíduo em estudo, obtivemos a média dos dois lados estudados nas variáveis comprimento, largura e espessura e realizamos os estudos a seguir com base nessa média.

\section{Características macroscópicas das pregas vocais quanto ao sexo}

Quanto ao comprimento das pregas vocais, observamos que houve diferença estatística $(p<0,001)$ entre os grupos masculino e feminino. Analisando a largura das pregas vocais, também obtivemos uma diferença estatística entre os grupos masculino e feminino $(p=0,04)$. Na análise da espessura, não houve diferença entre os grupos $(p=0,11)$. o Gráfico 2 mostra a comparação dos comprimentos, enquanto 0 Gráfico 3 evidencia as relações da largura e espessura quanto ao sexo.

\section{Correlação entre as características dos indivíduos $e$ as dimensões das pregas vocais}

O btivemos os resultados apresentados na Tabela 1, evidenciando uma forte correlação (coeficiente de Pearson maior que 0,75 ) entre a altura dos indivíduos e 0 comprimento das pregas vocais. Não encontramos esta correlação quando analisamos a largura e a espessura das pregas vocais.

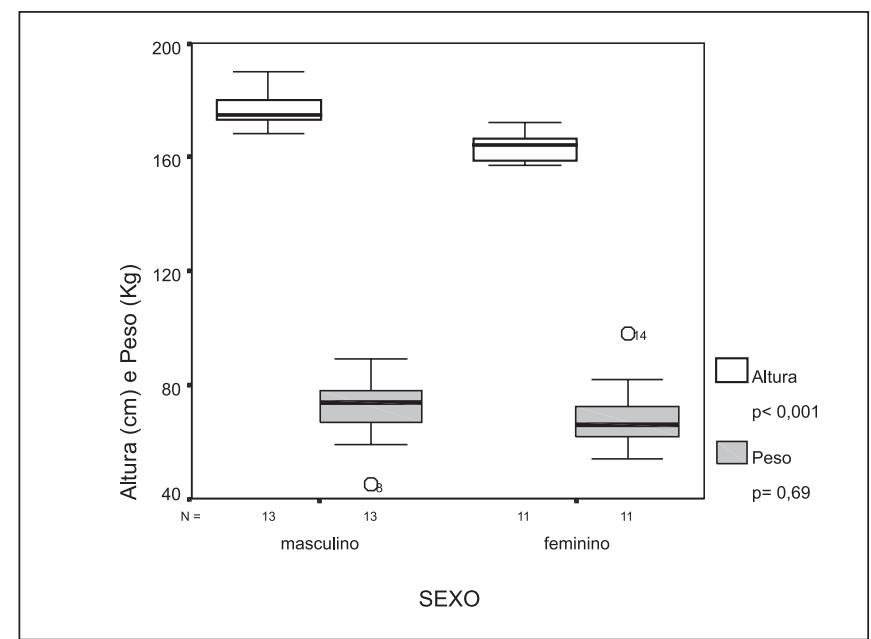

Gráfico 1. Comparação entre altura e peso conforme o sexo.

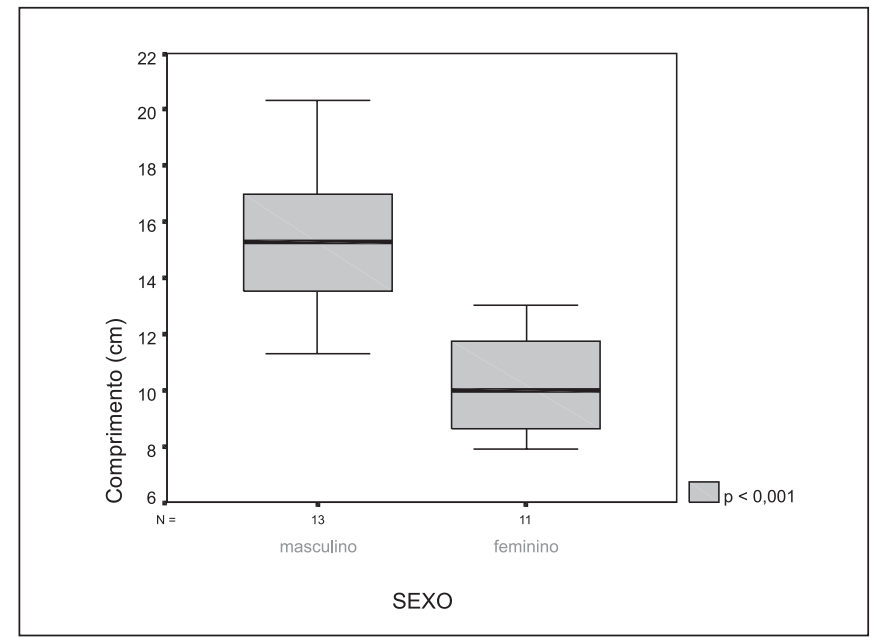

Gráfico 2. Comparação entre os comprimentos das pregas vocais quanto ao sexo.

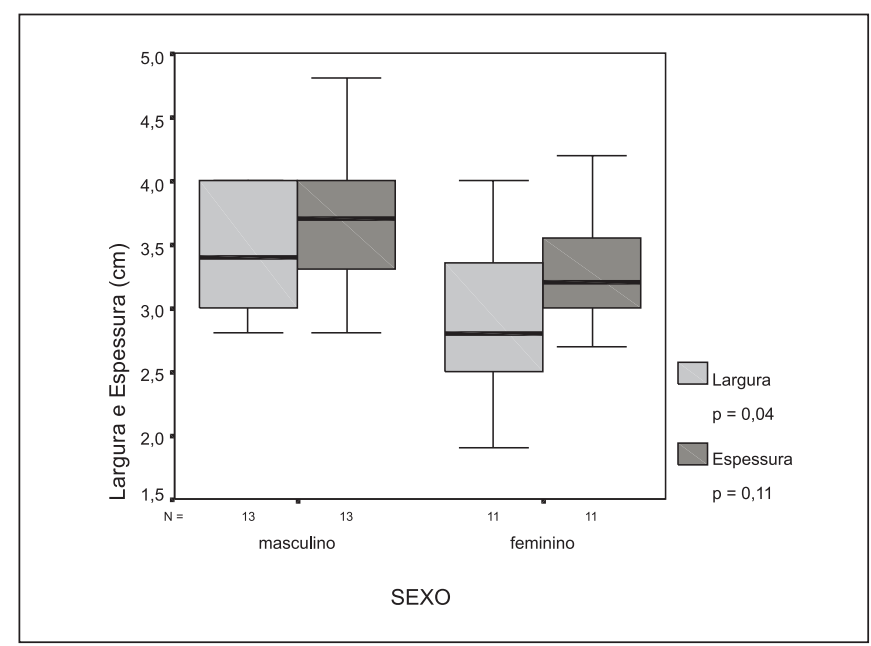

Gráfico 3. Comparação entre a largura e espessura das pregas vocais quanto ao sexo. 
Tabela 1. Tabela com o Coeficiente de Correlação de Pearson (r) e a significância estatística (p). *Correlação significante ao nível de 0,01.

\begin{tabular}{ccccccc}
\hline & \multicolumn{2}{c}{ Comprimento } & \multicolumn{2}{c}{ Largura } & \multicolumn{2}{c}{ Espessura } \\
& $\mathrm{r}$ & $\mathrm{p}$ & $\mathrm{r}$ & $\mathrm{p}$ & $\mathrm{r}$ & $\mathrm{p}$ \\
\hline altura & $0,76^{*}$ & $<0,001$ & 0,36 & 0,09 & 0,39 & 0,06 \\
peso & 0,24 & 0,27 & 0,00 & 0,99 & $-0,06$ & 0,78 \\
Idade & $-0,02$ & 0,93 & 0,47 & 0,83 & 0,37 & 0,08 \\
sexo & $0,77^{*}$ & $<0,001$ & 0,28 & 0,25 & 0,28 & 0,18 \\
\hline
\end{tabular}

\section{DISCUSSÃO}

Os estudos em cadáveres humanos, desde as detalhadas descrições de Andreas Vesalius na primeira metade do século XIV, têm nos ajudado a melhor compreender o corpo humano. Em especial na laringologia, os estudos anatômicos em laringes de cadáveres têm contribuído no entendimento das funções deste importante órgão. A laringe inicia seu desenvolvimento na terceira semana de vida intra-uterina, a partir de um prolongamento da faringe, de origem endodérmica. Apresenta já no terceiro mês de vida intra-uterina as características encontradas ao nascimento ${ }^{5}$. Após o nascimento, ocorrem diversas modificações nas configurações geométricas de todo o trato vocal. 0 comprimento da porção membranosa das pregas vocais quase duplica no primeiro ano de vida, atingindo, na idade adulta, 11,5 a $16 \mathrm{~mm}$ no homem e 8,0 a 11,5 mm na mulher. A porção cartilaginosa tem um crescimento bem mais discreto, atingindo 5,5 a 7,0 mm nos homens e 4,5 a 5,5 nas mulheres 6 . Ocorre um aumento progressivo do comprimento da parte intermembranácea das pregas vocais nas duas primeiras décadas de vida, com diferenciação evidente quanto ao sexo aos 15 anos de idade?

Uma dúvida que também nos motivou na realização deste estudo foi quanto ao encurtamento da porção membranosa das pregas vocais com o envelhecimento, em especial em indivíduos do sexo masculino ${ }^{8}$. No nosso estudo, contudo, não encontramos correlação entre a idade e 0 comprimento da porção membranosa das pregas vocais. A razão disto se deve possivelmente pela forma diferente de análise estatística empregada. Além disso, acreditamos que as alterações vocais decorrentes do envelhecimento estão mais relacionadas a mudanças microarquiteturais das pregas vocais ${ }^{9,10}$ do que com as modificações nas dimensões macroscópicas das pregas vocais.

A fim de obtermos dados com a maior precisão possível, utilizamos uma fita métrica colocada ao lado do corpo do indivíduo estudado que se encontrava em decúbito dorsal para a verificação da altura do indivíduo. No entanto, na medida de peso, utilizamos dados do prontuário do indivíduo, que é baseado nos dados coletados junto aos familiares, uma vez que o peso do indivíduo poderia está modificado pelas situações que o levaram ao óbito como edema, caquexia, desidratação ou ainda pelas modificações teciduais que ocorrem após a morte. Como a altura não varia nestas circunstâncias, esta medida é muito confiável.

$\mathrm{Na}$ análise estatística de nossos resultados, optamos pela aplicação do teste estatístico de Correlação de Pearson. Este teste não traduz relação de causa e efeito, porém nos mostra se há ou não correlação entre uma variável independente (altura, idade, sexo e peso) e as diversas variáveis dependentes testadas (dimensões das pregas vocais).

Os principais achados deste estudo preliminar foram a confirmação da hipótese de que existe uma correlação entre a altura do indivíduo e o comprimento de suas pregas vocais e a observação de que para um homem e uma mulher da mesma altura, as pregas vocais não apresentam o mesmo comprimento, mostrando que as diferenças do arcabouço laríngeo refletem numa diferença na glote membranosa.

\section{CONCLUSÕES}

Podemos concluir que houve correlação entre a altura e o comprimento das pregas vocais e não houve correlação da altura com a largura ou com a espessura das pregas vocais. Não encontramos correlação das dimensões das pregas vocais com o peso ou com a idade dos indivíduos. Observamos diferença estatística entre as pregas vocais de homens e mulheres quanto ao comprimento e a largura, o que não foi observado na espessura.

\section{REFERÊNCIAS BIBLIOGRÁFICAS}

1. Isshiki N, Tsuji DH, Sennes LU. Tireoplastias. $1^{\text {a }}$ ed. São Paulo, SP: Bios Comunicação e Editora; 1999.

2. Slavit DH. Phonosurgery in the elderly: a review. Ear Nose Throat J 1999;78:505-9, 12.

3. Tucker HM. Larryngeal framework surgery in the management of the aged larynx. Ann Otol Rhinol Laryngol 1988;97:534-6.

4. Lejune FE, Guice CE, Samuels, PM. Early experiences with vocal ligament tightening. Ann Otol Rhinol Laryngol 1983:92:475-7.

5. Behlau M. Voz - 0 Livro do Especialista. Rio de Janeiro, RJ: Revinter; 2001.

6. Aroson AE. Clinical Voice Disorders. $3^{\text {rd }}$ ed. New York, NY: BC Decker, 1990.

7. Kurita S, Nagata K, Hirano M. A comparative study of the layer structure of the vocal fold. In Bless D, Abbs J, eds. Vocal fold physiology contemporary research and clinical issues. San Diego, California: College-Hill; 1983.

8. Hirano M, Kurita S, Sakaguchi S. Ageing of the vibratory of human vocal folds. Acta Otolaryngol 1989;107:428-33.

9. Sato K, Hirano M. Age-related changes of elastic fibers in the superficial layer of the lamina propria of vocal folds. Ann Otol Rhinol Laryngol 1997;106:44-8.

10. Hammond TH, Gray SD, Butler J, Zhou R, Hammond E. Age- and gender-related elastin distribution changes in human vocal folds. Otolayngol Head Neck Surg 1998;119:314-22. 